\title{
Diversidade, produção e recepção
}

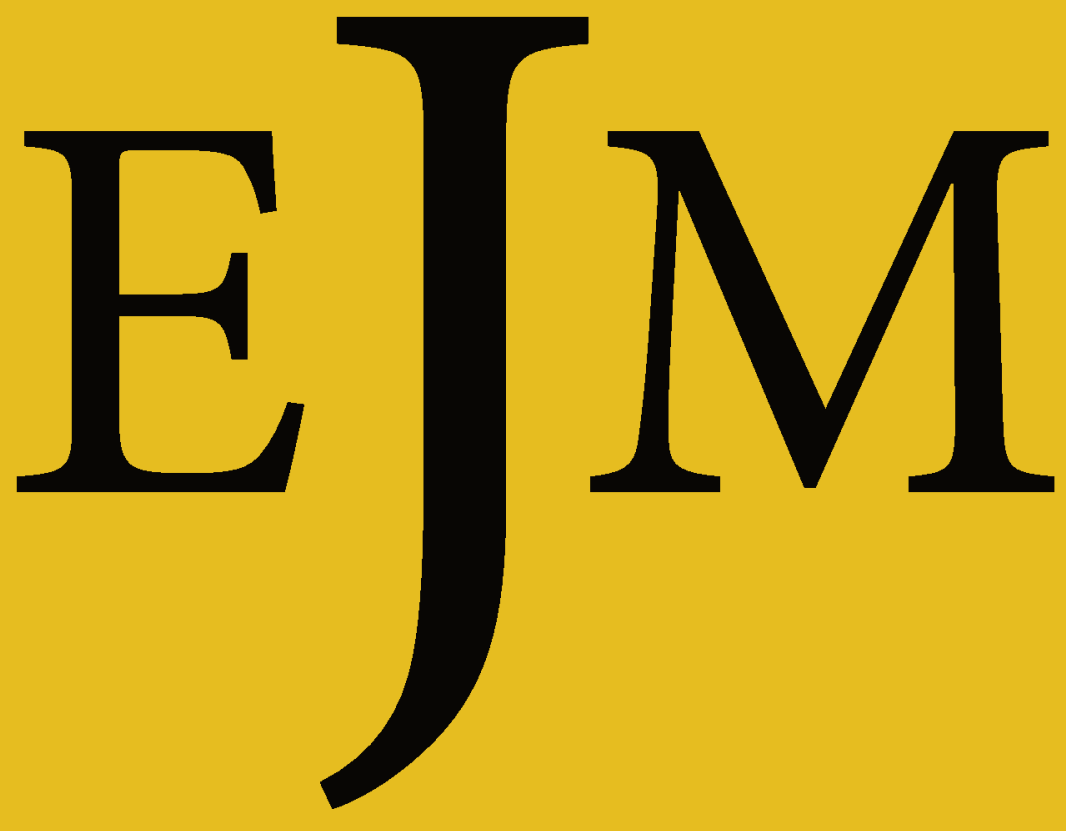

Revista Acadêmica Semestral Programa de Pós-Graduação em Jornalismo Universidade Federal de Santa Catarina 


\section{Universidade Federal de Santa Catarina}

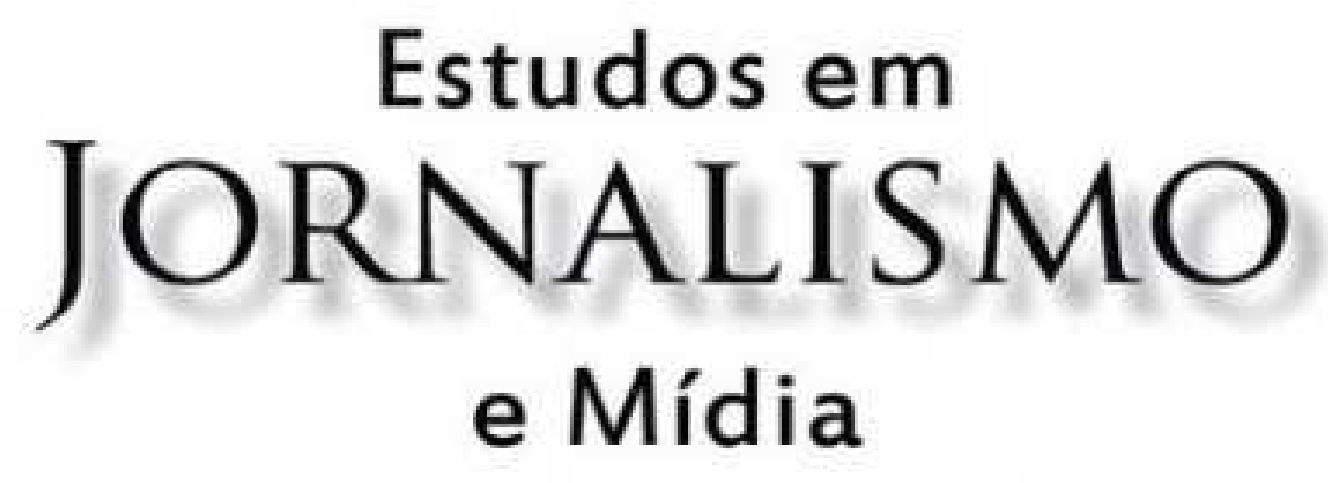

Volume 15 - № 2

Julho a Dezembro 2018

ISSNe 1984-6924

(c) $(1)(9)$ 


\title{
Universidade Federal de Santa Catarina
}

\author{
Reitor \\ Ubaldo César Balthazar \\ Pró-Reitor de Pesquisa \\ Sebastião Roberto Soares \\ Pró-Reitora de Pós-Graduação \\ Cristiane Derani \\ Centro de Comunicação e Expressão \\ Diretor \\ Arnoldo Debatin Neto \\ Departamento de Jornalismo \\ Chefe \\ Maria José Baldessar \\ Programa de Pós-Graduação em Jornalismo \\ Coordenadora \\ Carlida Emerim
}

Estudos em Jornalismo e Mídia - Volume 15, nº 2, Julho a Dezembro de 2018

Semestral

Revista Acadêmica Semestral On-line - Programa de Pós-Graduação em Jornalismo da Universidade Federal de Santa Catarina

$2^{\text {a }}$ edição

ISSNe 1984-6924

1. Jornalismo 2. Mídia I.Título

Universidade Federal de Santa Catarina

Programa de Pós-Graduação em Jornalismo

http://www.posjor.ufsc.br

Florianópolis - SC - Brasil

Fone: +55 48 3721-6610

Revista Estudos em Jornalismo e Mídia

http://periodicos.ufsc.br/index.php/jornalismo

E-mail: ejm@contato.ufsc.br 


\section{Estudos em \\ JORNALISMO \\ e Mídia}

Comissão Editorial: Daiane Bertasso e Terezinha Silva (editoras)

Projeto Gráfico e Capa: Sílvio da Costa Pereira e Ana Carolina Passos Entrevistador: William Robson Cordeiro Silva

Revisão: Ana Marta Moreira Flores (traduções), Dairan Paul, Edwin Carvalho, Ingrid Pereira de Assis, Janaíne Kronbauer dos Santos, Janara Nicoletti, Juliana Gobbi Betti, Karina Woehl de Farias, Magali Moser, Marli Vitali, Rafael Rangel Winch, Ricardo Torres, William Robson Cordeiro Silva.

Editoração e produção: Isabela Martins

Apoio de Produção: Sílvio da Costa Pereira e Equipe do Portal de Periódicos da UFSC

\section{Conselho Editorial}

Adriana Amado, UNLAM, Argentina

Aglair Bernardo, UFSC, Brasil

Alfredo Vizeu, UFPE, Brasil

Antonio Hohlfeldt, PUC-RS, Brasil

Ana Carolina Escosteguy, PUC-RS, Brasil

Ana Paula Goulart Ribeiro, UFRJ, Brasil

Basilio G. Monteiro, Saint John's University, EUA

Beatriz Becker, UFRJ, Brasil

Carlos Eduardo Franciscato, UFS, Brasil

Claudia Lago, ECA/USP, Brasil

Claudia Mellado Ruiz, Usach, Chile

Christa Berger, Unisinos, Brasil

Dione Oliveira Moura, UnB, Brasil

Dóris Fagundes Haussen, PUC-RS, Brasil

Edson Fernando Dalmonte, UFBA, Brasil

Eduardo Meditsch, UFSC, Brasil

Elton Antunes, UFMG, Brasil

Flávio Porcello, UFRGS, Brasil

Francisco José Castilhos Karam, UFSC, Brasil

Gislene Silva, UFSC, Brasil

Heloíza Herscovitz, California State Univ., EUA

Isabel Ferin Cunha, U. Coimbra, Portugal
Jacques Mick, UFSC, Brasil

João Canavilhas, UBI, Portugal

Jorge Pedro Sousa, Un. Fernando Pessoa, Portugal

Josenildo Luiz Guerra, UFS, Brasil

Juan Carlos Suárez Villegas, Un. Sevilla, Espanha

Kênia Beatriz Ferreira Maia, UFRN, Brasil

Lila Luchessi, UNRN, Argentina

Luiz Gonzaga Motta, UnB-UFSC, Brasil

Manuel Pinto, Universidade do Minho, Portugal

Marcos Palácios, UFBA, Brasil

Marcia Benetti, UFRGS, Brasil

Maria José Baldessar, UFSC, Brasil

Martín Becerra, UBA, Argentina

Nilson Lemos Lage, UFSC, Brasil

Paulo Bernardo Ferreira Vaz, UFMG, Brasil

Rosana Soares, USP, Brasil

Silvio Waisbord, George Washington Univ., EUA

Sonia Virgínia Moreira, UERJ, Brasil

Susana Herrera Damas, Univ. Carlos III, Espanha

Suzana Barbosa, UFBA, Brasil

Victor Gentilli, UFES, Brasil 